\title{
Etude, sur coupes histologiques, de la migration des larves de Syngamus trachea (Montagu 1811) Chapin 1925 au sein d'Eisenia foetida Sav.
}

\author{
par Anne-Michelle NOLST (1) \\ Laboratoire de Parasitologie ( $\mathrm{P}^{r} \mathrm{D}^{r} \mathrm{R}$. VAnBreuseghem), Université libre de Bruxelles, \\ 115, bd de Waterloo, Bruxelles, Belgique
}

\section{Résumé}

Le but de ce travail était d'étudier la migration des larves de Syngamus trachea à l'intérieur d'Eisenia foetida.

Nous avons pour cela infesté des vers immatures âgés de 15 jours ou de 2 à 3 mois avec des œufs embryonnés de syngames ayant bénéficié de 10 jours d'incubation à $25^{\circ} \mathrm{C}$, au moins.

Grâce à des coupes histologiques de vers fixés, après différents temps d'infestation, nous avons pu mettre certains faits en évidence.

L'éclosion des larves de $S$. trachea a lieu surtout dans la seconde moitié de l'intestin moyen d'E. foetida.

Les larves écloses traversent la paroi de l'intestin postérieur, pour pénétrer dans le coelome à ce niveau.

Elles envahissent alors rapidement les muscles du corps de leur hôte, dans les segments correspondant à l'intestin postérieur d'abord et dans tous les segments ensuite.

\section{Summary}

Histological study of Syngamus trachea (Montagu 1811) Chapin 1925 larves in Eisenia foetida Sav.

The migration of larvae of S. trachea in E. foetida has been investigated.

(1) Ces recherches ont bénéficié du soutien financier de l'Institut pour l'Encouragement de la Recherche Scientifique dans l'Industrie et l'Agriculture. 
Immature worms 15 days or 2 at 3 months old were infested with embryonated eggs of $S$. trachea incubated for 10 days at $25^{\circ} \mathrm{C}$.

Our observations demonstrate that the larvae of $S$. trachea hatch mainly in the second half of the middle intestine of E. foetida.

Liberated larvae penetrate the coelomic cavities through the wall of the posterior intestine.

From there, they invade rapidly the muscles of the worm's wall at the same level.

Finally, they dispers in the all musculature of their host.

\section{Introduction.}

Le rôle des vers de terre dans la propagation de la syngamose aviaire est connu depuis 1886, date à laquelle H. D. Walker suggère que Syngamus trachea fait partie de la faune intestinale de Lumbricus terrestris.

Pour P. A. Clapham (1934), le Lombricien Eisenia foetida est un hôte plus commun de $S$. trachea que $L$. terrestris. L'auteur décrit des larves de syngames du troisième stade, logées dans des kystes profondément enfoncés dans les muscles de l'Eisenia hôte.

E. L. Taylor $(1935,1938)$ confirme ces dernières observations. Pour cet auteur, le kyste proviendrait d'une réaction inhabituelle de la part de l'hôte et se formerait graduellement au cours du temps.

E. E. Wehr (1959) émet l'hypothèse que les larves de $S$. trachea ingérées par Helodrilus foetidus (syn. E. foetida) éclosent à l'intérieur de cet hôte, percent la paroi intestinale et s'enkystent dans la musculature.

Dans un travail précédent (A.-M. Nolst, 1971), nous décrivions quelques localisations de larves de $S$. trachea trouvées chez $E$. foetida maintenu expérimentalement, pendant 15 jours, en contact avec le milieu infestant. Nous relations en outre le fait qu'au début de l'infestation ( 24 premières heures), $98 \%$ des larves se trouvent dans les segments postérieurs des vers alors que 15 jours plus tard, 17 à $23 \%$ d'entre elles ont envahi les 45 premiers segments. En nous basant sur ces résultats, nous formulions l'hypothèse que les larves de syngames quittent le tube digestif au niveau des segments postérieurs de l'Eisenia hôte pour remonter en partie vers les régions antérieures en s'établissant dans la musculature.

Le présent travail a pour but d'éclairer la manière dont les larves de $S$. trachea se déplacent au sein d'E. foetida.

\section{Matériel et méthodes.}

Les œufs de $S$. trachea, isolés par flottaison, à partir de fientes de dindons infestés expérimentalement, sont mis en incubation dans de l'eau de ville à $25^{\circ} \mathrm{C}$, pendant au moins 10 jours.

Les $E$. foetida utilisés sont élevés, dès leur éclosion, dans du terreau autoclavé addi- 
tionné de Cegomin. Le Cegomin est le tourteau résiduel des fruits du cacaoyer; il favorise la croissance et la maturation d'E. foetida (H. Herlant-Meewis et P. Van Gansen, 1964).

Pour infester les vers, nous les plaçons dans un cristallisoir contenant un peu d'eau de ville très riche en œufs embryonnés infestants de syngames. Ces animaux sont mis à jeun 1 ou 2 jours avant l'infestation.

Nous préciserons, dans nos expériences, l'âge des vers utilisés. Toutefois, il s'agit généralement d'animaux immatures, âgés de 15 jours ou de 2 à 3 mois.

Les vers étudiés sont fixés, entiers, au Bouin-Hollande. Ils sont ensuite découpés en 3 ou 4 tronçons dont nous effectuons des coupes longitudinales de 7 microns. Il est ainsi possible d'avoir une image de la localisation des larves de syngames dans un ver au moment choisi.

\section{Résultats expérimentaux.}

Le but de ces expériences était de mettre en évidence le lieu où les larves de $S$. trachea infestantes éclosent dans le tube digestif d' $E$. foetida, l'endroit où elles pénètrent dans le cœlome et le trajet qu'elles effectuent ensuite.

1. Eclosion des larves de S. trachea, dans le tube digestif d'E. foetida.

Nous avons tenté de préciser la partie du tube digestif où a lieu l'éclosion des larves infestantes de $S$. trachea à l'intérieur d' $E$. foetida.

$1^{\circ}$ Nous avons pu surprendre, sur les coupes histologiques d'une dizaine de vers, 24 larves de syngames au moment de leur éclosion; 18 d'entre elles se trouvaient dans la seconde moitié de l'intestin moyen et dans les premiers segments de l'intestin postérieur, les autres étant disséminées un peu partout dans le tube digestif.

Cette constatation nous a fait penser que c'était peut-être dans la seconde moitié de l'intestin moyen que la plupart des larves de syngames éclosaient.

$2^{\circ}$ Comme nous avions remarqué, d'autre part, qu'il y avait beaucoup plus de larves écloses dans l'intestin postérieur et le rectum que dans les régions antérieures du tube digestif, nous avons calculé le pourcentage des larves écloses par rapport au nombre total de larves de syngames trouvées chez 10 Eisenia, dans les 35 premiers segments du tube digestif, d'une part, et dans l'intestin postérieur et le rectum, d'autre part. Il s'agit des régions antérieure et postérieure à la zone où la majorité des éclosions avaient été observées. Les résultats sont consignés dans le tableau suivant.

\begin{tabular}{|c|c|c|}
\hline & $\begin{array}{l}35 \text { premiers seg- } \\
\text { ments (bouche-pre- } \\
\text { mière moitié intes- } \\
\text { tin moyen) }\end{array}$ & $\begin{array}{l}\text { Intestin postérieur- } \\
\text { rectum }\end{array}$ \\
\hline $\begin{array}{l}\text { Nombre total de larves } \ldots \ldots \ldots \ldots \ldots \ldots \ldots \\
\text { Nombre de larves non écloses } \ldots \ldots \ldots \ldots \ldots \ldots \\
\text { Nombre de larves écloses } \ldots \ldots \ldots \ldots \ldots \ldots \\
\text { Pourcentage de larves écloses } \ldots \ldots \ldots \ldots \ldots\end{array}$ & $\begin{array}{r}171 \\
167 \\
4 \\
2\end{array}$ & $\begin{array}{r}118 \\
70 \\
48 \\
40\end{array}$ \\
\hline
\end{tabular}

Pourcentage d'éclosions de larves de $S$. trachea dans différentes régions du tube digestif d'E. foetida 
Le pourcentage des larves écloses de syngames est donc, en effet, beaucoup plus élevé dans l'intestin postérieur et le rectum que dans les 35 premiers segments du tube digestif des Eisenia.

Les photos 1, 2 et 3 de la planche 1 montrent respectivement des œufs embryonnés, une larve en train d'éclore et des larves écloses de $S$. trachea dans le tube digestif d'E. foetida.

Il ressort de ces quelques observations que les larves de $S$. trachea éclosent surtout dans la seconde moitié de l'intestin moyen d'E. foetida.

\section{Envahissement d'E. foetida par les larves de S. trachea.}

Les expériences décrites ci-après ont été faites dans le but de voir où les larves de syngames quittent le tube digestif des vers pour entrer dans leur cœlome.

$1^{\circ}$ Nous avons étudié la distribution de 143 larves de $S$. trachea chez $10 \mathrm{E}$. foetida de 15 jours, après 8 heures d'infestation, en dehors de la lumière de leur tube digestif. Quatre-vingt-quinze pour cent d'entre elles étaient situées dans les segments coïncidant avec l'intestin postérieur.

$2^{\circ}$ De plus, la cinquantaine de larves que nous avons trouvées dans la paroi du tube digestif de 20 vers, après 4 ou 8 heures d'infestation, étaient toutes situées dans l'épithélium ou dans les couches sous-jacentes à l'épithélium de l'intestin postérieur. La manière dont certaines d'entre elles étaient orientées nous a amenée à penser que les larves de syngames traversent la paroi de l'intestin postérieur d'E. foetida pour pénétrer dans son coelome, comme le montrent les photos 4 (planche 1), 1 et 2 (planche 2).

C'est donc, au niveau de l'intestin postérieur, que les larves de $S$. trachea percent la paroi intestinale en direction du cœlome d'E. foetida.

3. Trajet effectué par les larves de $\mathrm{S}$. trachea, à partir du colome des segments de l'intestin postérieur d'E. foetida.

Ces observations ont été faites afin de déterminer ce que deviennent les larves de syngames, une fois qu'elles ont quitté le tube digestif de leur hôte.

$1^{\circ}$ Nous avons tout d'abord étudié la distribution des larves de syngames au niveau des segments coïncidant avec l'intestin postérieur des vers observés précédemment (cf. $2,1^{\circ}$ ).

Les 136 larves trouvées à ce niveau étaient réparties comme suit:

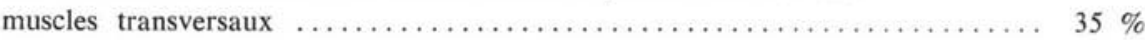

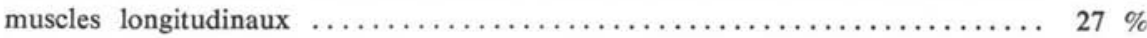

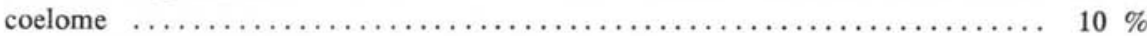

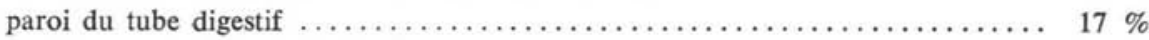

Les larves de syngames atteignent donc rapidement les muscles de la paroi des vers, puisqu'après 8 heures d'infestation, $63 \%$ d'entre elles y sont déjà situées. La photo 3 de la planche 2 montre une larve en train de pénétrer dans les muscles longitudinaux, av niveau de l'intestin postérieur.

Planche 1. - Eufs et larves de $S$. trachea chez $E$. foetida. Photo 1: Gufs embryonnés dans l'intestin moyen. Photo 2: Larve en train d'éclore dans l'intestin moyen. Photo 3: Larves écloses dans l'intestin postérieur. Photo 4: Larve pénétrant dans l'épithélium de l'intestin postéríeur. c., coelome; e., épithélium cilié de l'intestin postérieur; t.d., lumière du tube digestif; 1., larve 


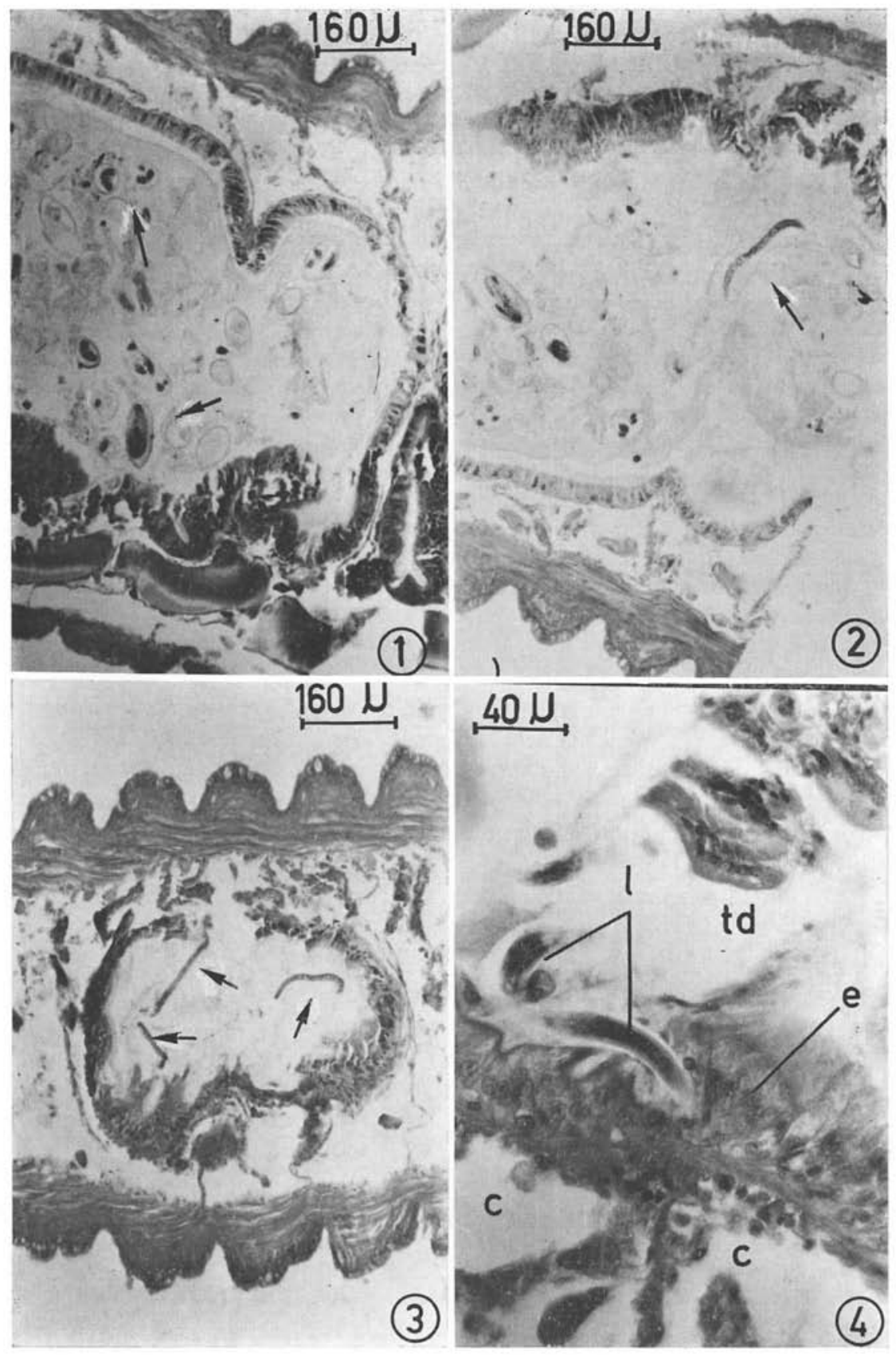


$2^{\circ}$ Ensuite, comme nous avions très vite remarqué qu'au cours du temps les larves se dispersent dans tous les segments du ver, nous avons examiné la localisation de 101 larves de syngames trouvées chez 5 vers de 15 jours, après 48 heures d'infestation, en dehors de la lumière de leur tube digestif ( $90 \%$ des parasites étaient alors dans la musculature). La répartition des larves le long des vers était la suivante: $46 \%$ dans les segments s'étendant de la bouche à l'intestin moyen inclus et $16 \%$ dans les segments correspondant au rectum. Il ne restait donc plus que $39 \%$ des parasites au niveau de l'intestin postérieur.

$\mathrm{Si}$ nous comparons ces derniers résultats avec ceux que nous avions obtenus, après 8 heures d'infestation, il apparaît clairement qu'une partie des parasites se disperse dans toute la musculature des vers qui les hébergent. Eisenia.

$3^{\circ} \mathrm{Il}$ reste à déterminer comment les larves de syngames se déplacent le long des

Les expériences que nous poursuivons actuellement montrent que, antérieurement et postérieurement à leur zone de pénétration, on retrouve les parasites dans les muscles longitudinaux avant de les retrouver dans les muscles transversaux de leur hôte. Ce fait semble indiquer qu'ils effectuent leurs migrations en voyageant dans la couche musculaire longitudinale.

D'ailleurs, sur les coupes de vers infestés, lez larves apparaissent très généralement disposées parallèlement aux fibres musculaires, que ce soit dans les muscles transversaux ou dans les muscles longitudinaux (cf. photo 4, planche 2). Il semble donc logique de penser que les larves effectuent leurs déplacements dans ces derniers.

\section{Discussion et conelusions.}

Les quelques résultats que nous venons de décrire éclairent le processus de migration des larves de $S$. trachea au sein d'E. foetida.

L'éclosion des larves a lieu dans le tube digestif des vers qui les ont ingérées. Elle se fait surtout dans la seconde moitié de l'intestin moyen. Or, cette zone correspond à la fin de la portion sécrétrice du tube digestif d'E. foetida (P. Van Gansen, 1962). Il sera donc intéressant de voir s'il y a une action des sécrétions du jabot, du gésier, de l'intestin antérieur ou de l'intestin moyen sur l'éclosion des larves de S. trachea.

Les larves de $S$. trachea quittent le tube digestif d'E. foetida pour pénétrer dans son cœlome, en traversant la paroi de l'intestin postérieur. En effet, aucune larve n'a été repérée à un autre endroit de la paroi intestinale et, après 8 heures d'infestation, $95 \%$ des parasites sont situés à ce niveau.

Les larves de $S$. trachea envahissent directement les muscles d'E. foetida au niveau de leur zone de pénétration dans le cœlome (intestin postérieur), puisque, après 8 heures d'infestation, $63 \%$ d'entre elles s'y trouvent déjà.

Enfin, les larves de $S$. trachea se dispersent dans toute la musculature d'E. foetida: après 48 heures d'infestation, il ne reste plus que $39 \%$ des parasites au niveau de l'intestin postérieur. Les expériences que nous poursuivons actuellement nous amènent

Planche 2. - Larves de $S$. trachea chez $E$. foetida. Photo 1: dans l'épithélium de l'intestin postérieur. Photo 2: traversant la paroi de l'intestin postérieur. Photo 3: pénétrant dans les muscles longitudinaux. Photo 4: dans les muscles longitudinaux. m.l., muscles longitudinaux; m.t., muscles transversaux ; p., peau 


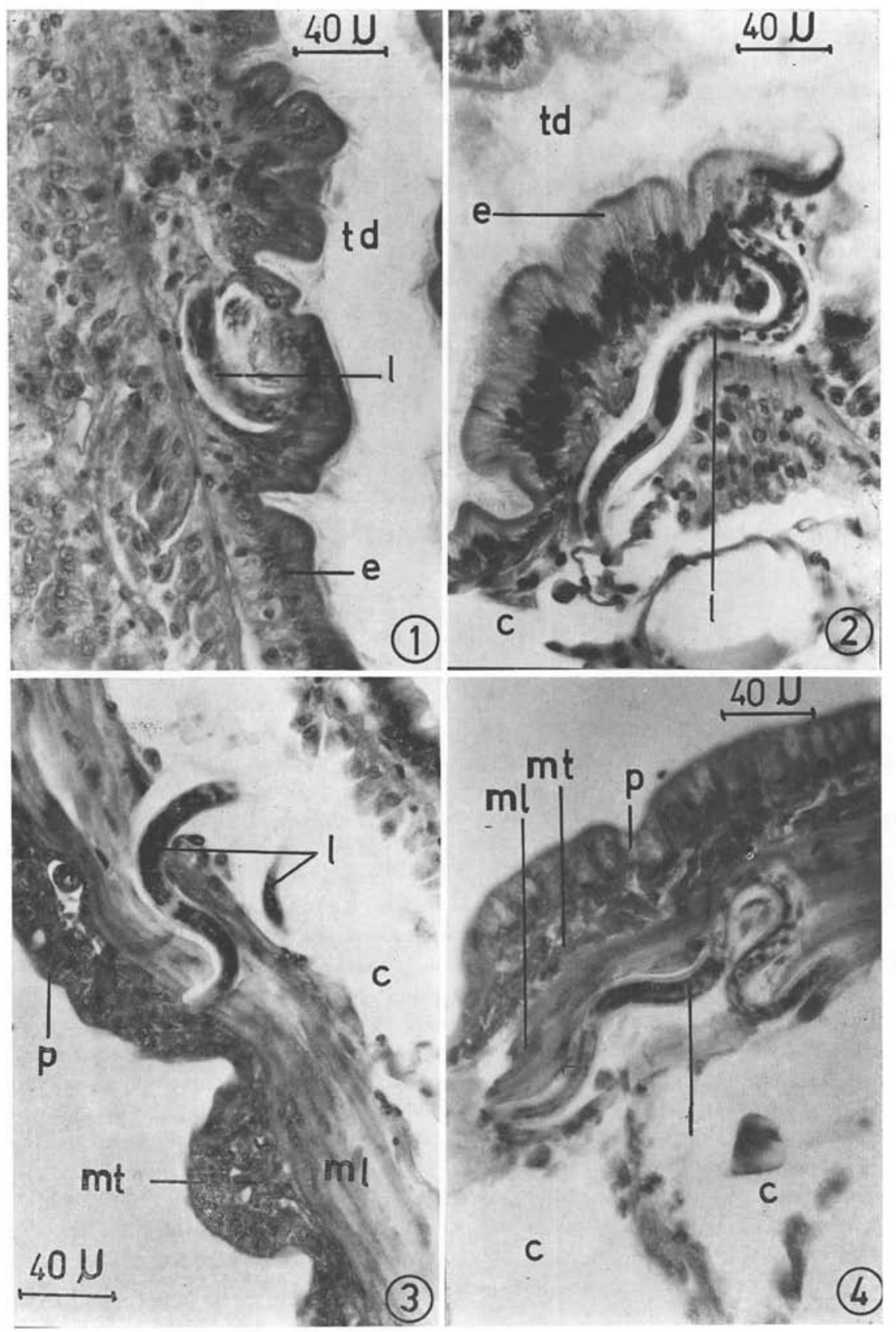


à penser que ces derniers se déplaceraient entre les fibres musculaires des muscles longitudinaux.

Il existe quelques travaux à propos de l'observation de coupes de vers de terre infestés par des larves de Nématodes. Nous en citerons deux qui traitent de problèmes parallèles à notre sujet.

Par exemple, R. W. Allen (1949) dit avoir observé sur des coupes d'Allolobo. phora caliginosa infesté par Capillaria annulata, des larves de ce Nématode dans les muscles longitudinaux des vers. Dans ce cas, ce sont les larves du premier stade qui sont infestantes pour le ver de terre dans le tube digestif duquel elles éclosent.

A. J. Probert (1969) décrit les migrations des larves de Metastrongylus spp. à l'intérieur d'E. foetida. Ce sont les larves du premier stade qui se développent dans les glandes calciques et les sinus de la paroi du jabot, du gésier et de l'intestin antérieur. Elles gagnent ensuite le vaisseau dorsal et les cœurs des vers parasités.

Les migrations des larves de $S$. trachea à l'intérieur du ver de terre n'ont encore jamais fait l'objet d'études. Nous avons pu, grâce à des coupes d' $E$. foetida, infesté et fixé à des moments précis, mettre en évidence certaines étapes de ces migrations, depuis le moment où les œufs embryonnés contenant les larves du troisième stade de $S$. trachea sont ingérés par les vers jusqu'à l'établissement de ces larves dans les muscles de leur hôte.

\section{Bibliographie}

Allen (R. W.), 1949. - Studies on the Life history of Capillaria annulata (Mollen, 1958) Cram, 1926. J. Parasit., 35 (suppl.), 35.

Clapham (P. A.), 1934. - Experimental studies on the transmission of gapeworm (Syngagamus trachea) by Earthworms. Proc. Roy. Soc. Lond. (B), 115, 18-29.

Herlant-Meewis (H.), Van Gansen (P.), 1964. - Influence des milieux d'élevage expérimentaux sur la croissance et la sexualité du Lombricien Eisenia foetida Sav. Ann. Soc. Roy. Zool., Belgique, 94, 213-226.

Nolst (A. M.), 1971. - Infestation d'Eisenia foetida Sav. par Syngamus trachea (Montagu, 1811) Chapin, 1925. Ann. Parasit. hum. comp., 46, 257-264.

Probert (A. J.), 1969. - Morphological and histochemical studies on the larval stages of Metastrongylus spp. (lungworm of swine) in the Earthworm intermediate host Eisenia foetida Sav., 1826. Parasitology, 59 (2), 269-277.

TAYLOR (E. L.), 1935. - Syngamus trachea. The longevity of the infective larvae in the earthworms, slugs and snails as intermediate host. J. Comp. Path., 57, 149-165.

-, 1938. - An extension to the known longevity of gapeworm infection in Earthworms and Snails. Vet. Journ., 94, 327.

VAN Gansen (P.), 1962. - Structures et fonctions du tube digestif du Lombricien Eisenia foetida Sav. Ann. Soc. Roy. Zool. Belgique, 93 (1).

WALKER (H. D.), 1886. - The gapeworm of fowls (Syngamus trachealis) : the Earthworm Lumbricus terrestris, its original host. Bull. Buff. Soc. Nat. Sc., 5 (2), 47-71.

WeHR (E. E.), 1959. - In diseases of Poultry, pp. 747-750. H. E. Biester and L. H. Sharte : The iowa state college press, 1959. 